\title{
Series: Cardiovascular outcome trials for diabetes drugs Degludec and DEVOTE
}

\author{
MILES FISHER
}

\begin{abstract}
DEVOTE (Trial Comparing Cardiovascular Safety of Insulin Degludec versus Insulin Glargine in Patients with Type 2 Diabetes at High Risk of Cardio-vascular Events) was an FDAmandated cardiovascular outcome trial and was the first and at present the only - completed trial comparing two insulins. DEVOTE compared insulin degludec and insulin glargine (U100) in 7,637 people with type 2 diabetes with established cardiovascular disease, chronic kidney disease, or both, and older diabetic patients with increased cardiovascular risk. DEVOTE demonstrated non-inferiority for major cardiovascular events (MACE), a composite of death from cardiovascular causes, non-fatal myocardial infarction and non-fatal stroke, with a pre-specified non-inferiority margin of 1.3. At 24 months the mean $\mathrm{HbA}_{1 \mathrm{c}}$ was similar in the two groups. Mean fasting plasma glucose was significantly lower in the degludec group than in the glargine group, and prespecified severe hypoglycaemia was also significantly lower in the degludec group. DEVOTE satisfied the FDA cardiovascular safety requirements for new antidiabetic therapies.

Br J Diabetes 2021;21:113-115
\end{abstract}

Key words: diabetes, cardiovascular outcome trial, degludec, glargine

\section{Introduction}

Between 2008 and 2020 the licensing requirements for new antidiabetic drugs for the FDA in the USA usually required a dedicated cardiovascular outcome trial (CVOT) to satisfy criteria for cardiovascular safety. ${ }^{1}$ The primary endpoints were atherosclerotic events, either major cardiovascular events (MACE; a composite of cardiovascular death, non-fatal myocardial infarction or non-fatal stoke), or major cardiovascular events plus hospitalisation for unstable angina. The study design could either be the new antidiabetic drug versus placebo or the new drug versus an active comparator, and most CVOTs used a placebo comparator group. Dedicated CVOTs have been performed for several drugs within the dipeptidyl pep-

Address for correspondence: Professor Miles Fisher Department of Diabetes, Endocrinology \& Clinical Pharmacology, Glasgow Royal Infirmary, 84 Castle Street, Glasgow G4 OSF, UK

E-mail: miles.fisher@ggc.scot.nhs.uk

https://doi.org/10.15277/bjd.2021.302 tidase-4 (DPP-4) inhibitor, glucagon-like peptide-1 (GLP-1) receptor agonist and sodium-glucose co-transporter-2 (SGLT2) inhibitor drug classes. The outcomes for the first seven of these CVOTs have previously been reviewed in this series, describing the primary endpoint plus important secondary outcomes and subsequent publications of data from subgroups and post hoc analyses. ${ }^{2-8}$ The ORIGIN trial, completed in 2012, was an investigator-initiated CVOT comparing glargine with placebo in subjects with type 2 diabetes or prediabetes. ${ }^{9}$ ORIGIN used a study design that was similar to FDA-mandated CVOTS. DEVOTE was the first FDA-mandated CVOT of an insulin and compared insulin degludec with glargine. ${ }^{10}$

\section{Background}

Degludec is an ultralong-acting once daily basal analogue insulin. It was recommended for approval by the European Medicines Agency in 2012 in formulations of 100 units/mL (U100) and 200 units $/ \mathrm{mL}$ (U200). It was submitted to the FDA at a similar time, but the FDA advisory committee thought that there was a consistent and persistent signal of excess cardiovascular risk associated with degludec relative to comparators, based on meta-analysis of cardiovascular events from the Phase 2 and Phase 3 development programme. The FDA requested a dedicated double-blind CVOT with glargine as the comparator. Degludec was approved for use in the USA following a resubmission in 2015, based on interim analysis of data from DEVOTE, and the DEVOTE study was completed in 2017.

\section{DEVOTE}

A paper describing the design and baseline characteristics of DEVOTE was published in 2016. ${ }^{11}$ The principal DEVOTE results were presented in 2017 at a meeting of the American Diabetes Association with simultaneous publication in the New England Journal of Medicine. ${ }^{10}$ The design of the study and key baseline characteristics are shown in Box 1.

In DEVOTE, the primary outcome of MACE, a composite of cardiovascular death, non-fatal myocardial infarction and nonfatal stroke, occurred in $8.5 \%$ of the degludec group and $9.3 \%$ of the glargine group, demonstrating non-inferiority with a prespecified upper boundary of the $95 \%$ confidence interval less than 1.3 , but not superiority. This satisfied the FDA cardiovascular safety criteria from 2008 where a post-marketing trial was required to definitely show that the upper bound of the twosided $95 \%$ confidence interval for the estimated risk ratio was less than 1.3. There was no difference in an expanded primary 


\section{Box 1 Key features of DEVOTE 10,11}

- DEVOTE compared degludec with glargine in a treat-to-target trial in 7,637 subjects with type 2 diabetes for 24 months

- The target was a pre-breakfast self-measured blood glucose concentration of 4.0-5.0 mmol/L, with a target of 5.0-7.0 $\mathrm{mmol} / \mathrm{L}$ for vulnerable patients

- The mean age of subjects was 65 years with a mean duration of diabetes 16 years

- Mean baseline $\mathrm{HbA}_{1 \mathrm{c}}$ was $8.4 \%$ (68 $\mathrm{mmol} / \mathrm{mol}$ ) falling to $7.5 \%$ $(58 \mathrm{mmol} / \mathrm{mol}$ ) at 24 months

- $63 \%$ of subjects were aged $\geq 50$ years and had established cardiovascular disease, $16 \%$ chronic kidney disease and $21 \%$ established cardiovascular disease plus chronic kidney disease. $14 \%$ of subjects were aged $\geq 60$ years and had risk factors for cardiovascular disease. $12 \%$ of subjects had baseline heart failure.

- $16 \%$ of subjects were not on insulin at baseline, $38 \%$ were on basal insulin only and $46 \%$ were on basal-bolus insulin, including bolus only insulin and premixed insulin. $60 \%$ of subjects were on metformin, 29\% sulfonylureas, 12\% DPP-4 inhibitors, $8 \%$ GLP-1 receptor agonists, 2\% SGLT2 inhibitors

Figure 1. 24-month event rates (in \%) comparing degludec and glargine for major adverse cardiovascular events (MACE), total mortality, cardiovascular mortality, non-fatal myocardial infarction, non-fatal stroke and hospitalisation for heart failure (HFH). There were no statistically significant differences between the two groups

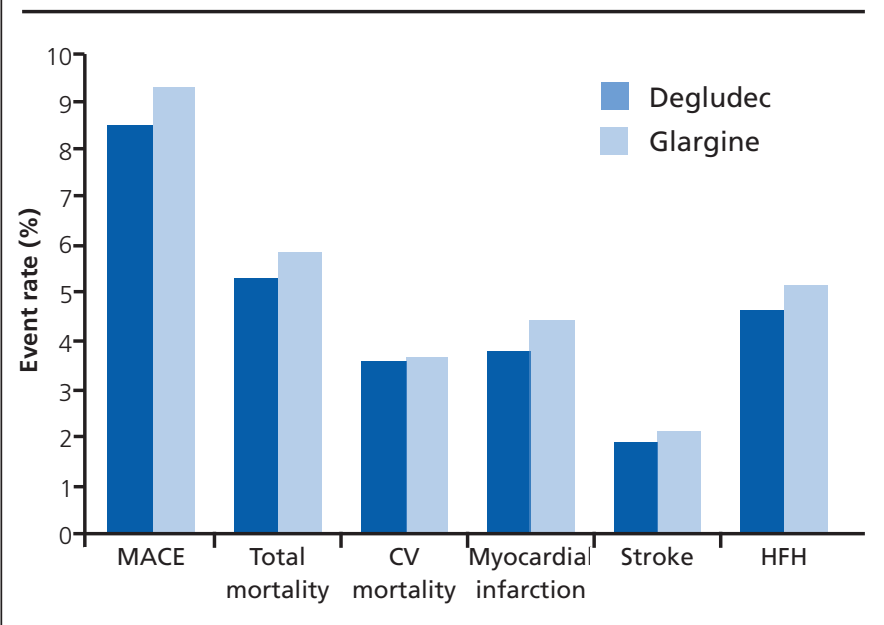

composite of MACE plus hospitalisation for unstable angina, and there were no significant differences in all-cause mortality, cardiovascular death, non-fatal myocardial infarction or non-fatal stroke (Figure 1, Box 2). At 24 months the $\mathrm{HbA}_{1 \mathrm{c}}$ concentrations were similar in the two groups, but fasting plasma glucose was significantly lower in the degludec group at $7.1 \pm 3.1 \mathrm{mmol} / \mathrm{L}$ versus $7.5 \pm 3.2 \mathrm{mmol} / \mathrm{L}$ in the glargine group. Pre-specified adjudicated severe hypoglycaemia, defined as an episode requiring the assistance of another person to actively administer carbohydrate or glucagon, or take corrective action, was significantly reduced in the degludec group (Box 2), as was nocturnal severe hypoglycaemia. Body weight increase by around $2 \mathrm{~kg}$ in both groups.
Box 2 Results of the DEVOTE trial

Principal result

- No difference in major adverse cardiovascular events (MACE)10

Other results from DEVOTE

- Severe hypoglycaemia was significantly reduced in the degludec group with 280 events in 187 patients versus 472 events in 252 patients in the glargine group ${ }^{10}$

- The strongest risk factor for hospitalisation for heart failure (HHF) was prior heart failure, and the risk of HHF increased significantly within a week of an episode of severe hypoglycaemia'2

- There was a non-significant increase in MACE in subjects who had experienced severe hypoglycaemia, and no temporal relationship between severe hypoglycaemia and MACE ${ }^{15}$

- Patients with increasing age had higher risks of cardiovascular death and all-cause mortality, with non-significant increases in MACE and severe hypoglycaemia ${ }^{16}$

- The DEVOTE population was divided into quartiles of hypoglycaemia risk and, in the group with the highest risk, severe hypoglycaemia was associated with a high incidence of MACE and all-cause mortality, and those treated with degludec had a lower incidence of MACE ${ }^{17}$

- The risks of MACE, cardiovascular death and all-cause mortality increased with worsening glomerular filtration rate ${ }^{18}$

- A history of heart failure was a risk factor for chronic kidney disease, as was a history of hepatic impairment ${ }^{19}$

- Long-term modelling suggested that degludec was cost effective from the prospective of England's National Health Service in basal-bolus regimens for patients with type 2 diabetes at high cardiovascular risk ${ }^{20}$

\section{Other results from DEVOTE}

Further publications from DEVOTE are detailed in Box 2. There has been much clinical interest in hospitalisation for heart failure as a secondary outcome in CVOTs. In DEVOTE, heart failure was not an adjudicated outcome and heart failure events were analysed using the standardised MedDRA query definition which is restricted to specific terminology, symptoms, signs and investigational features that are pathognomonic for cardiac failure. ${ }^{12}$ As might be expected, there was no difference in hospitalisation for heart failure events between the treatment groups. Prior heart failure was the strongest predictor of future heart failure events. Interestingly, shortly after an episode of severe hypoglycaemia, the risk of hospitalisation for heart failure increased significantly.

\section{Discussion}

The DEVOTE trial demonstrated no significant differences in cardiovascular events between insulin degludec and insulin glargine. Glargine was chosen as an active comparator in a double-blind trial as several of the phase 3 studies with degludec used openlabel glargine as the comparator, and cardiovascular endpoint data were available for glargine from the ORIGIN trial. ${ }^{9}$ Although the ORIGIN trial was similar to an FDA-mandated trial, there were several key differences: in ORIGIN many subjects had prediabetes or newly diagnosed diabetes and the mean duration of diabetes for people with prior diabetes was 5 years. ORIGIN compared glargine with placebo, and cancers were also compared in addition to cardiovascular outcomes, microvascular outcomes, incident diabetes, hypoglycaemia and weight. The 


\section{Key messages}

- DEVOTE was the first FDA-mandated cardiovascular outcome trial comparing two insulins

- DEVOTE compared insulin degludec with insulin glargine and there were no differences in cardiovascular events

- Using a treat-to-target approach, degludec was associated with fewer episodes of severe hypoglycaemia than glargine

reduction in severe and nocturnal hypoglycaemia comparing degludec with glargine U100 that was seen in the DEVOTE trial mirrors similar differences in hypoglycaemic events that were demonstrated in the SWITCH 1 and SWITCH 2 trials, which compared rates of symptomatic hypoglycaemia with degludec versus glargine U100 in randomised double-blind trials in people with type 1 and type 2 diabetes, respectively. ${ }^{13,14}$

Conflict of interest The author reports personal fees from Allergan, Amgen, AstraZeneca, Bayer, Boehringer Ingelheim, Eli Lilly, Mylan, NAPP, Novo Nordisk, Sanofi and from Servier outside the submitted work.

\section{Funding None.}

\section{References}

1. Chong WH, Yanoff LB, Andraca-Carrera E, Thanh Hai M. Assessing the safety of glucose-lowering drugs - a new focus for the FDA. N Engl J Med 2020;383:1199-202. https://doi.org/10.1056/NEJMp2004889

2. Fisher M. Saxagliptin and SAVOR-TIMI 53. Br J Diabetes 2019;19:34-6. https://doi.org/10.15277/bjd.2019.213

3. Fisher M. Alogliptin and EXAMINE. Br J Diabetes 2019;19:133-5. https://doi.org/10.15277/bjd.2019.230

4. Fisher M. Lixisenatide and ELIXA. Br J Diabetes 2020;20:52-4. https://doi.org/10.15277/bjd.2020.241

5. Fisher M. Sitagliptin and TECOS. Br J Diabetes 2020;20:55-7. https://doi.org/10.15277/bjd.2020.242

6. Fisher M. Empagliflozin and EPA-REG OUTCOME. Br J Diabetes 2020; 20:138-41. https://doi.org/10.15277/bjd.2020.254

7. Fisher M. Liraglutide and LEADER. Br J Diabetes 2020;20:142-4. https://doi.org/10.15277/bjd.2020.267

8. Fisher M. Semaglutide and SUSTAIN-6. Br J Diabetes 2021;21 (in press).

9. ORIGIN Trial Investigators. Basal insulin and cardiovascular and other outcomes in dysglycemia. N Engl J Med 2012;367:319-28. https://doi.org/10.1056/NEJMoa1203858

10. Marso SP, McGuire DK, Zinman B, et al, for the DEVOTE Study Group. Efficacy and safety of degludec versus glargine in type 2 diabetes. N Engl J Med 2017;377:723-32. https://doi.org/10.1056/NEJMoa1615692

11. Marso SP, McGuire DK, Zinman B, et al. Design of DEVOTE (Trial Comparing Cardiovascular Safety of Insulin Degludec vs Insulin Glargine in Patients with Type 2 Diabetes at High Risk of Cardio-vascular Events) - DEVOTE 1. Am Heart J 2016;179:175-83. https://doi.org/10.1016/j.ahj.2016.06.004

12. Pratley RE, Husain M, Lingvay I, et al, on behalf of the DEVOTE Study Group. Heart failure with insulin degludec versus glargine U100 in patients with type 2 diabetes at high risk of cardiovascular disease: DEVOTE 14. Cardiovasc Diabeto/ 2019;18:156. https://doi.org/10.1186/s12933019-0960-8

13. Lane W, Bailey TS, Gerety G, et al. Effect of insulin degludec vs insulin glargine U100 on hypoglycaemia in patients with type 1 diabetes. The SWITCH 1 randomized clinical trial. JAMA 2017;318:33-44. https://doi.org/10.1001/jama.2017.7115

14. Wysham C, Bhargava A, Chaykin L, et al. Effect of insulin degludec vs insulin glargine U100 on hypoglycaemia in patients with type 2 diabetes. The SWITCH 2 randomized clinical trial. JAMA 2017;318:45-56. https://doi.org/10.1001/jama.2017.7117

15. Pieber TR, Marso SP, McGuire DK, et al, on behalf of the DEVOTE Study Group. DEVOTE 3: temporal relationship between severe hypoglycaemia, cardiovascular outcomes and mortality. Diabetologia 2018; 61:58-65. https://doi.org/10.1007/s00125-017-4422-0

16. Pratley RE, Emerson SS, Franek E, et al, on behalf of the DEVOTE Study Group. Cardiovascular safety and lower severe hypoglycaemia of insulin degludec versus insulin glargine U100 in patients with type 2 diabetes aged 65 years or older: results from DEVOTE (DEVOTE 7). Diabetes Obes Metab 2019;21:1625-33. https://doi.org/10.1111/dom.13699

17. Heller S, Lingvay I, Marso SP, et al, Devote Study Group. Risk of severe hypoglycaemia and its impact in type 2 diabetes in DEVOTE. Diabetes Obes Metab 2020;22:2241-7. https://doi.org/10.1111/dom.14049

18. Amod A, Buse JB, McGuire DK, et al. on behalf of the DEVOTE Study Group. Glomerular filtration rate and associated risks of cardiovascular events, mortality, and hypoglycemia in patients with type 2 diabetes; secondary analysis (DEVOTE 11). Diabetes Ther 2020;11:53-70. https://doi.org/10.1007/s13300-019-00715-x

19. Amod A, Buse JB, McGuire DK, et al, on behalf of the DEVOTE Study Group. Risk factors for kidney disorders in patients with type 2 diabetes at high cardiovascular risk: an exploratory analysis (DEVOTE 12). Diab Vasc Dis Res 2020;17:1479164120970933. https://doi.org/10.1177/ 1479164120970933

20. Pollock RF, Valentine WJ, Marso SP, et al, on behalf of the DEVOTE Study Group. Long-term cost-effectiveness of insulin degludec versus insulin glargine U100 in the UK: evidence from the basal-bolus subgroup of the DEVOTE trial (DEVOTE 16). Appl Health Econ Health Policy 2019; 17:615-27. https://doi.org/10.1007/s40258-019-00494-3 